\title{
Effect of obstacle position in the flow of sheep through a narrow door
}

\author{
Iker Zuriguel, ${ }^{1}$ Jorge Olivares, ${ }^{1}$ José M. Pastor, ${ }^{1}$ César Martín-Gómez, ${ }^{2}$ Luis M. Ferrer, ${ }^{3}$ \\ Juan J. Ramos, ${ }^{3}$ and Angel Garcimartín ${ }^{1}$ \\ ${ }^{1}$ Departamento de Física, Facultad de Ciencias, Universidad de Navarra, E-31080 Pamplona, Spain \\ ${ }^{2}$ Departamento de Construcción, Instalaciones y Estructuras, Escuela Técnica Superior de Arquitectura, \\ Universidad de Navarra, E-31080 Pamplona, Spain \\ ${ }^{3}$ Departamento de Patología Animal, Facultad de Veterinaria, Universidad de Zaragoza, Miguel Servet 177, 50013 Zaragoza, Spain
}

(Received 17 June 2016; published 1 September 2016)

\begin{abstract}
In a recent work [Phys. Rev. E 91, 022808 (2015)] it was reported that placing an obstacle in front of a gate has a beneficial effect in the flow of sheep through it. Here, we extend such results by implementing three different obstacle positions. We have observed that the flow is improved in two cases, while it worsens in the other one; the last instance happens when the obstacle is too close to the door. In this situation, the outcomes suggest that clogging develops between the doorjamb and the obstacle, contrary to the cases when the obstacle is farther, in which case clogging always occurs at the very door. The effectiveness of the obstacle (a strategy put forward to alleviate clogging in emergency exits) is therefore quite sensitive to its location. In addition, the study of the temporal evolution of the flow rate as the test develops makes evident a steady behavior during the entire duration of the entrance. This result is at odds with recent findings in human evacuation tests where the flow rate varies over time, therefore challenging the fairness of straightforward comparisons between pedestrian behavior and animal experimental observations.
\end{abstract}

DOI: 10.1103/PhysRevE.94.032302

\section{INTRODUCTION}

The behavior of human crowds trying to pass through a bottleneck when evacuating a building has been a topic which has risen notable interest in the past years [1-6]. Unfortunately, such emergency evacuations are hard to study for three main reasons. First, real emergency evacuations cannot be rehearsed at will and the data available from them is usually limited. Second, due to ethical concerns, experiments in controlled environments cannot put people at risk, so the competitiveness seen in real emergencies cannot be properly recreated. Finally, computer simulations offer great insight and are effective when planning emergency exits, but there are rules embedded in the programs that are not completely validated and, therefore, the extent to which conclusions can be drawn from them is limited. In past years, understanding the flow of crowds in competitive conditions has received increased attention in the field of pedestrian dynamics, so finding novel ways to approach this situation are welcomed. In this sense, it has been proposed that evacuation experiments with animals can provide an insight into human conduct [7].

In this context, many of the reported observations of animals have been carried out with ants [8-12], but noteworthy differences are salient when it comes to establishing analogies with human behavior. Ants seem to maneuver as a unified entity, working collectively to support the colony. Perhaps for this reason, it is believed that they do not interact competitively and do not show phenomena like jamming [8] or clogging [12], although this idea has been recently challenged [13]. An alternative to ants is the use of mice $[14,15]$. These have the advantage of being readily available as they are a standard test animal in other scientific fields. In addition, mice are able to remember and learn from previous situations, so they can adopt sensible strategies. In such works, two different approaches were employed to drive the mice towards the exit: a rising level of water (which mice do not like) was implemented in [14], whereas the smoke of burning joss sticks was used in [15]. In both cases, the authors report flow intermittencies which, in the case of [15], are surely due to highly competitive behavior and clogging in front of the door. In this latter work the existence of "faster is slower" (FIS) behavior was observed. This phenomenon relates to the fact that the evacuation might become less efficient as the pressure exerted by pedestrians at the door increases, and was first reported by Helbing and co-workers [1]. Subsequently, it was further developed by the same group and many others [16-19] to the point that it has become a standard signature of pedestrian dynamics when passing through narrow doors. Nevertheless, FIS has only recently been experimentally demonstrated for pedestrians [20] and it has been argued that it may constitute a general feature of the flow of many particle systems through constrictions, including inert grains and active matter besides pedestrians [21].

Another scenario in which resemblances among different multiparticle systems has been found is the placement of an obstacle in front of the exit [3,9,16,22-31]. Indeed, it has been shown that in such diverse systems as inert grains, ants, mice, and sheep, the presence of the obstacle can improve the flow rate due to the prevention of clogging. This is, in turn, caused by a reduction in the pressure near the outlet which is related to a density decrease resulting from the appearance of an empty region after the obstacle. Still, a thorough study about the effect of the obstacle position on the evacuation procedures has not been carried out experimentally, although numerical simulations [23] and experiments with inert particles discharged from a silo [25-27] are available.

In this manuscript, we aim to fill this gap and improve the understanding of the effect that the obstacle position has on the flow properties of sheep passing through a narrow door in a competitive manner. To this end we have performed experiments with three different obstacle positions, evidencing that there is an optimum location: if the obstacle is too 
far, its effect is weak as the small pressure reduction is not able to cause a notable improvement; if the obstacle is too close, clogs start to appear between the doorjamb and the obstacle, as previously evidenced in granular silos [25] and in numerical simulations [24]. In addition, in this work we will take advantage of the large amount of data collected to unveil whether the flow of sheep through bottlenecks displays any transient behavior as already observed in humans [32,33].

\section{SETUP}

We collected our data at a farm where sheep herds are kept and where we could observe the animals in their daily routines [30]. The sheep were young mothers of the same age and similar physical dimensions, the width of their hip being about $35 \mathrm{~cm}$. The whole flock consisted on more than one thousand sheep of Rasa Aragonesa breed. From this, a subset of 80 to 90 sheep was kept apart, and these were the animals we studied. Every morning, the farmer opens the door of the barn, which has a width of $96 \mathrm{~cm}$, and the sheep quickly enter the barn in order to eat. This is part of the farm everyday procedure, so we are observing the animals during their regular routines. Every 20 to 40 days, a new batch of approximately 80 to 90 sheep is drafted into the farm replacing the previous herd. We studied each herd for the first 10 to 20 days without the obstacle, and the last 10 to 20 days with the obstacle at a fixed position. In this work, we present data of three different herds, and each one of them was employed to test one obstacle position. From now on we will refer to herds $\mathrm{A}, \mathrm{B}$, and $\mathrm{C}$ to the flocks for which the obstacle was placed at 60,80 , and $100 \mathrm{~cm}$ from the door, respectively. Table I details the amount of runs and the number of data points (number of sheep registered) for each case, with and without an obstacle.

The cylindrical obstacle is a section of concrete drainpipe with a diameter of $114 \mathrm{~cm}$ and it is higher than the sheep, so that it blocks their view of the door when standing behind it. The obstacle was placed at a centered position from the door and the distance is measured from the center of the door to the closest point of the obstacle.

The sheep observation was performed by means of two cameras. These have been placed on both sides of the door in order to record the evacuation process from the top. The obtained videos were stored in a standard video surveillance system which registered two channels at 25 frames per second. This procedure was devised with the intention of facilitating the farmer daily tasks, as he had just to switch on the power

TABLE I. Summary of the experimental observations, where for each situation is given the number of runs, the total number of sheep recorded, and the average time interval between the passage of two consecutive sheep $\langle\Delta t\rangle$.

\begin{tabular}{|c|c|c|c|c|c|c|}
\hline & Herd A & Herd A & Herd B & Herd B & Herd C & Herd C \\
\hline Obstacle position & None & $60 \mathrm{~cm}$ & None & $80 \mathrm{~cm}$ & None & $100 \mathrm{~cm}$ \\
\hline Number of runs & 10 & 15 & 20 & 24 & 10 & 12 \\
\hline $\begin{array}{l}\text { Total number of } \\
\text { passage times }\end{array}$ & 830 & 1245 & 1708 & 1895 & 840 & 1008 \\
\hline$\langle\Delta t\rangle(\mathrm{s})$ & 0.32 & 0.37 & 0.30 & 0.27 & 0.37 & 0.32 \\
\hline
\end{tabular}

supply of the video system, which then automatically starts the recording without any further operation. Finally, every 30 to 40 days, we replaced the video surveillance hard disk and brought it to the laboratory where the videos were stored and analyzed.

The videos obtained from the cameras inside the barn are processed to create a space-time diagram as the one shown in Fig. 1 of [30]. Since the interest of the experiment is to count the time at which each sheep crosses the door, the line at which we count the sheep is drawn at a distance of around 1.5 sheep body lengths from the door. It is not done directly at the door because clogging can happen there. The diagram is built by horizontally stacking the selected line of pixels in a sequential manner for each frame of the video. Therefore, the horizontal dimension is the distance along the line and the vertical dimension is time. The sampling is accomplished with a MATLAB ${ }^{\circledR}$ program along a straight line parallel to the door and two circular segments that are centered at the door side ends. The thickness of the line is of 5 pixels, which increases the temporal resolution of the spatiotemporal diagram by a corresponding factor. Finally, the heads of the sheep are manually marked in each spatiotemporal diagram, and subsequently the passage time of each animal is straightforwardly obtained. This type of procedure has been proven to be relatively insensitive to small changes, so that small variations in the line position, the number of pixels of the line, or the part of the sheep body that is chosen as a reference to measure the passage time do not affect noticeably the results [34]. The temporal resolution achieved in the instant at which each animal crosses the line is about $0.05 \mathrm{~s}$.

\section{EXPERIMENTAL RESULTS}

With the data obtained from the spatiotemporal diagrams, the time at which each sheep crosses the door can be plotted against the number of animals $(\mathrm{N})$ that have already entered the barn. In Fig. 1 we represent one plot for each herd, where the times corresponding to all the rehearsals are plotted along with the mean time (calculated by averaging the passage time for each ordinal of the sheep crossing the door). Note that an obstacle position was investigated for each herd so both, the data for the scenario with and without obstacle, are plotted in each case.

The first noticeable property of these plots is that there exist visible differences among repetitions performed in the same conditions. Focusing for instance in herd B, even though the mean time needed to complete the herd passage is overall lower when there is an obstacle placed at $80 \mathrm{~cm}$, there are some runs without obstacle which were completed in a shorter time than other trials with the obstacle. This result stresses the importance of repeating the experiments several times in the same conditions before reaching any definitive conclusion. The effect of the obstacle can only be described in statistical terms. Furthermore, we can see that when the obstacle is at 80 or $100 \mathrm{~cm}$, the mean passing times are lower than when there is no obstacle. On the contrary, when the obstacle is placed at $60 \mathrm{~cm}$, the mean passing times are higher than when there is no obstacle.

In order to further investigate this behavior, in Fig. 2 we report the instantaneous flow $(J)$ calculated as the inverse of 

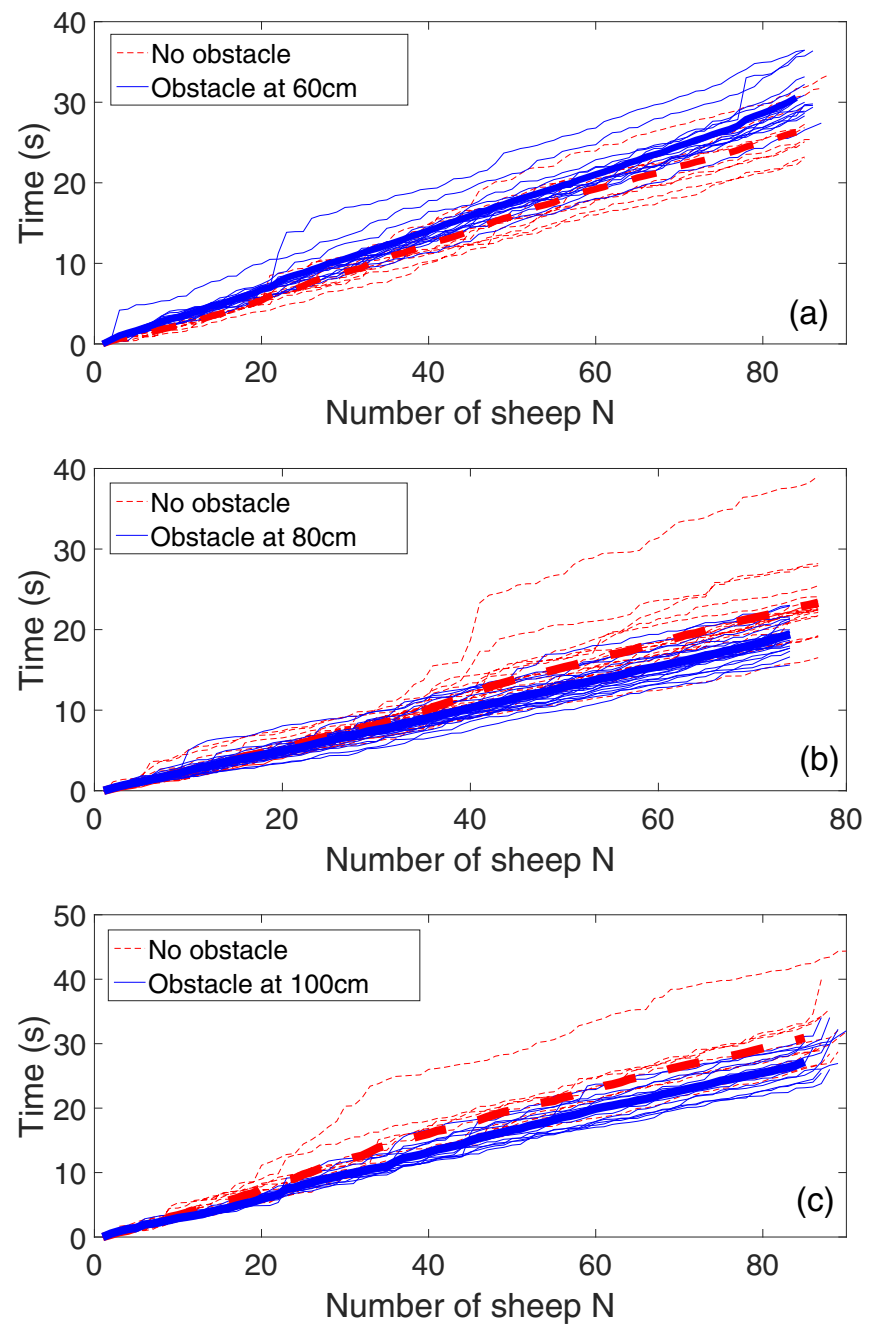

FIG. 1. Passage times, representing the number of individuals that have entered the barn vs time of (a) herd A, (b) herd B, and (c) herd C. The thin red dashed lines represent the trials without obstacle and the thin blue solid lines the tests with the obstacle at the positions indicated in legends. The thick lines are the averages of the passage times for each ordinal of the entering sheep: the solid blue line is for the cases where an obstacle is present, while the dashed red line is for the cases without obstacle.

the derivative of the data plotted in Fig. 1, that is $J=d N / d t$. Of course, this calculation increases the noise, so we have chosen to calculate $J$ as a moving average for windows of 10 sheep. In agreement with Fig. 1, we observe that the instantaneous flow when there is an obstacle at 80 or $100 \mathrm{~cm}$ is higher than when there is no obstacle, while the opposite happens with an obstacle at $60 \mathrm{~cm}$. In addition, the results of Fig. 2 allow us to confirm that this behavior is fulfilled over the whole process. More importantly, it can also be seen that -in all the cases - the instantaneous flow is rather constant throughout the whole transit of the herd (no transient is manifested at the beginning or the end of the process). This is opposed to what was recently observed with pedestrians $[32,33]$ where a clear transient, both at the beginning and at the end of the evacuation drill, was identified.
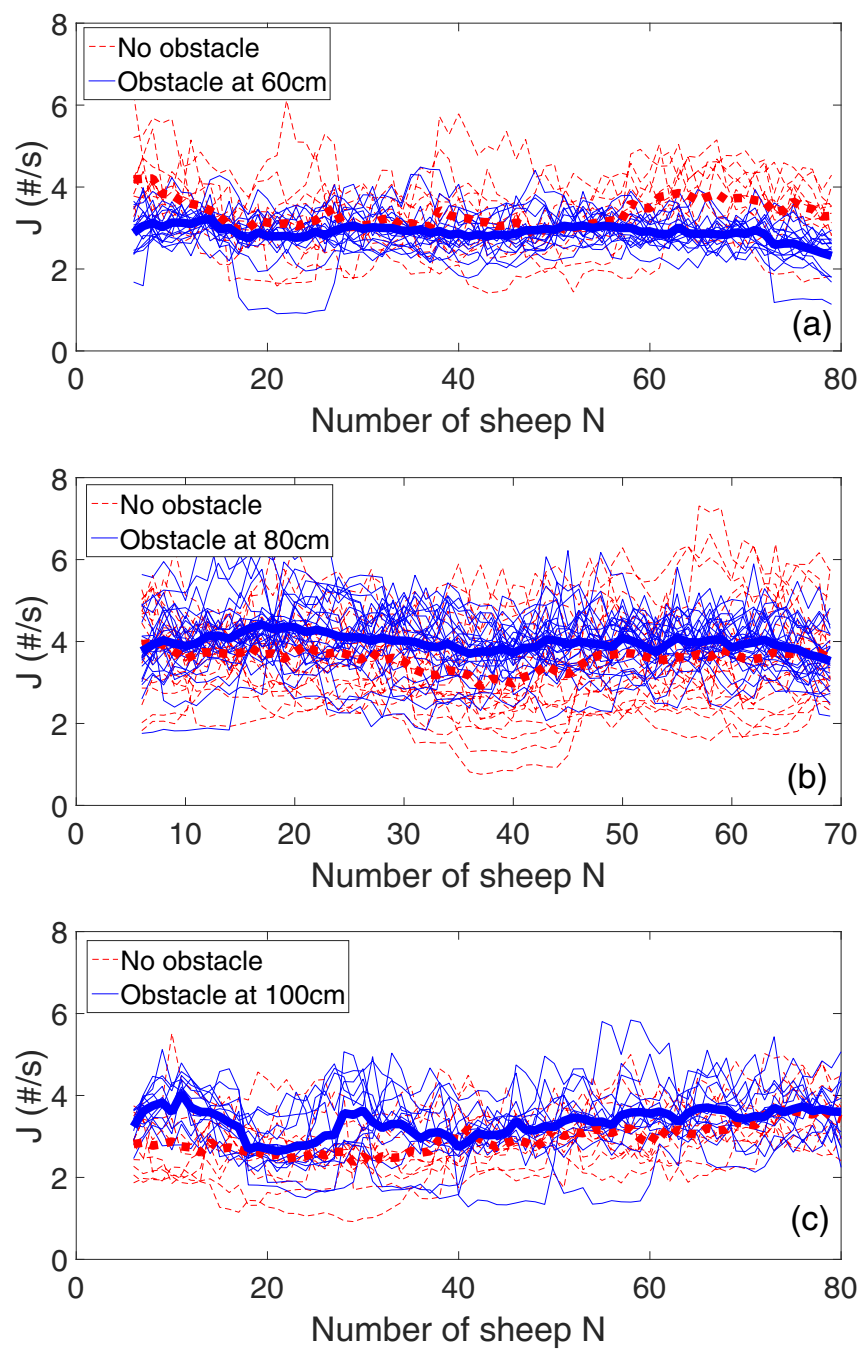

FIG. 2. Instantaneous flow rate for the same tests reported in Fig. 1. The instantaneous flow is calculated as the moving average for the derivative $d N / d t$ in a window of 10 consecutive sheep. Thin and thick lines, both solid and dashed, represent the same cases as in Fig. 1.

Apart from looking at global magnitudes (such as flow rate or total transit time), we can gain further insight of the flow properties by looking at the passage time between consecutive individuals $(\Delta t)$. To this end, and provided the apparent absence of transients, we grouped the results of all the rehearsals performed in the same conditions. The average values are reported in Table I indicating, once more, that the obstacle reduces the passage time when it is placed at 80 or $100 \mathrm{~cm}$, and increases it when placed at $60 \mathrm{~cm}$. As it is known that the distribution of $\Delta t$ is not Gaussian [28], a nonparametric test must be used to determine whether these central descriptors are equal (null hypothesis) or not. We have performed a permutation test, which is the following. Let $N_{0}$ be the number of passage times $\Delta t_{0}$ without an obstacle and $N_{1}$ the number of passage times $\Delta t_{1}$ with an obstacle, for a given herd. First we obtain a set of random permutations of $\left\{1,2, \ldots, N_{0}+N_{1}\right\}$ and reorder $\left\{\Delta t_{0}, \Delta t_{1}\right\}$ according to the indexes of the permutation. Then we calculate $d m$, the difference of the mean of the first $N_{0}$ data and the mean 


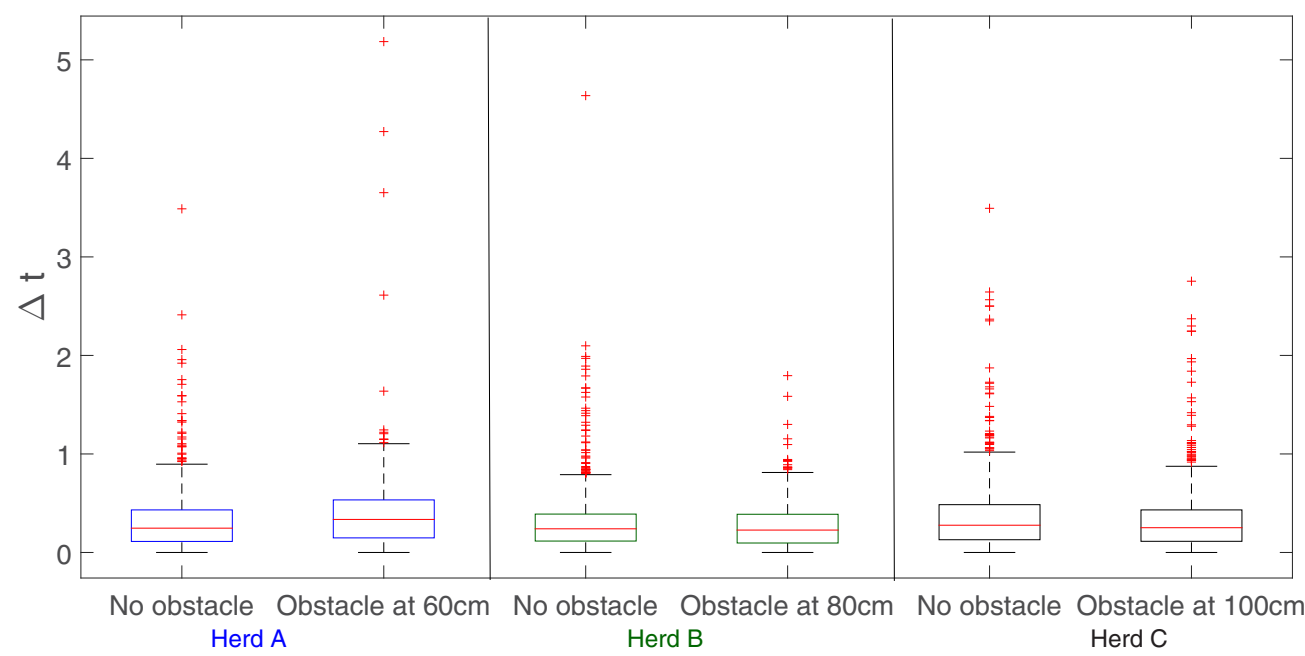

FIG. 3. Box-and-whiskers plot of the six experiments performed. The bottom and top of the boxes are the first and third quartiles for each experiment while the horizontal (red) lines indicate the second quartiles (the median values). The whiskers represent the lowest datum within 1.5 the interquartile range of the lower quartile, and the highest datum within 1.5 the interquartile range of the upper quartile. Outliers are marked with red crosses. In order to compare different cases, we have randomly chosen the same number of sheep for each case, i.e., 830 animals (which corresponds to the minimum of all the sets).

of the last $N_{1}$ data. This $d m$ is our statistic. If we perform this operation a large number of times, the distribution of $d m$ will tend to a normal. Then, the $p$ value is the portion of the area below the normal distribution of $d m$ bigger than the difference among the actual average of the data $\left\langle\Delta t_{0}\right\rangle-\left\langle\Delta t_{1}\right\rangle$ (multiplied by two to account for two tails). We have done this procedure for $10^{5}$ random permutations, and we obtained the following $p$ values for the difference of the averages between the case with and without an obstacle: $p=2 \times 10^{-4}$ (herd A), $p=3 \times 10^{-4}$ (herd B), and $p=0.005$ (herd C). Therefore, we can reject the null hypothesis that the averages come from the same distribution with a confidence level of $95 \%$. Noteworthy, we have compared the averages of the three cases without obstacle and obtained that only for herd A and B the null hypothesis cannot be rejected. On the contrary, herd $\mathrm{C}$ average times seem to be different than those of A and B.

Having said that, it is important to recall that the mean passage time is not the best indicator to characterize the flow properties as the distribution of such a variable could display extreme events [28]. A representation that gives an idea of such distributions is the box plot, as shown in Fig. 3. Clearly the medians are all very similar although, in agreement to what it was shown before, it can be perceived that the presence of the obstacle at 80 or $100 \mathrm{~cm}$ slightly reduces the median, and enlarges it when placed at $60 \mathrm{~cm}$. More significatively, the whiskers reveal a reduction of the data dispersion towards high values of $\Delta t$ when the obstacle is placed at 80 or $100 \mathrm{~cm}$, and an increase of such dispersion for the $60 \mathrm{~cm}$ case. Consistent with this observation is the fact that the outliers are notably affected by the presence of the obstacle and its position. In summary, from the box plot we learn that the median values of the distributions are rather insensitive to the obstacle position which, however, has a marked influence in the appearance of high values of $\Delta t$, i.e., long lasting clogs.

From these results, it becomes evident the need to investigate the distribution probability function (PDF) of $\Delta t$ for each particular scenario. In Fig. 4 we display the comparison between the probability density functions of each herd with and without the obstacle. Although not identical, the shape of the PDF is very similar regardless of the placement of the obstacle. This relates to the similarity obtained in the values of both means and medians. Indeed, this kind of linear plot is suitable to observe differences for small values of $\Delta t$ which, from what can be appreciated, are rather insensitive to the presence of the obstacle. Nonetheless, it should be pointed out that the obstacle at $60 \mathrm{~cm}$ seems to induce a noticeable reduction of the amount of small values of $\Delta t$, in agreement with the increase of the mean and median values.

A representation that seems more suitable to capture the differences appreciated in the box plot (which lie primarily in the upper outliers) is the complementary cumulative distribution function (also called reliability function in engineering, and survival function in life sciences) as shown in Fig 5. Such graphs stress differences in the tails, being specially convenient to analyze long lasting clogs which, by the way, could be the most dramatic danger in an evacuation. Notably, Fig. 5 shows that the obstacle at $80 \mathrm{~cm}$ leads to a extraordinary reduction of long lasting clogs (note the logarithmic scale). By placing the obstacle a bit further $(100 \mathrm{~cm})$, the same effect is obtained but it becomes considerably attenuated. Finally, for the obstacle at 60 $\mathrm{cm}$ a strange behavior is revealed. Indeed, the obstacle seems to reduce the amount of clogs lasting from 1 to $2 \mathrm{~s}$, but those larger than this quantity augment unexpectedly. Interestingly, this scenario is the first situation in which we observe that the distribution of clogging times of grains, sheep, or pedestrians, when passing through bottlenecks, is not compatible with a power law decay [28,30,32].

Even though this behavior is still far from understood, we can offer a plausible explanation based in qualitative observations. When the obstacle is too close to the door, sheep start clogging between the doorjamb and the obstacle rather than at the very door, as it is the case when the obstacle is 

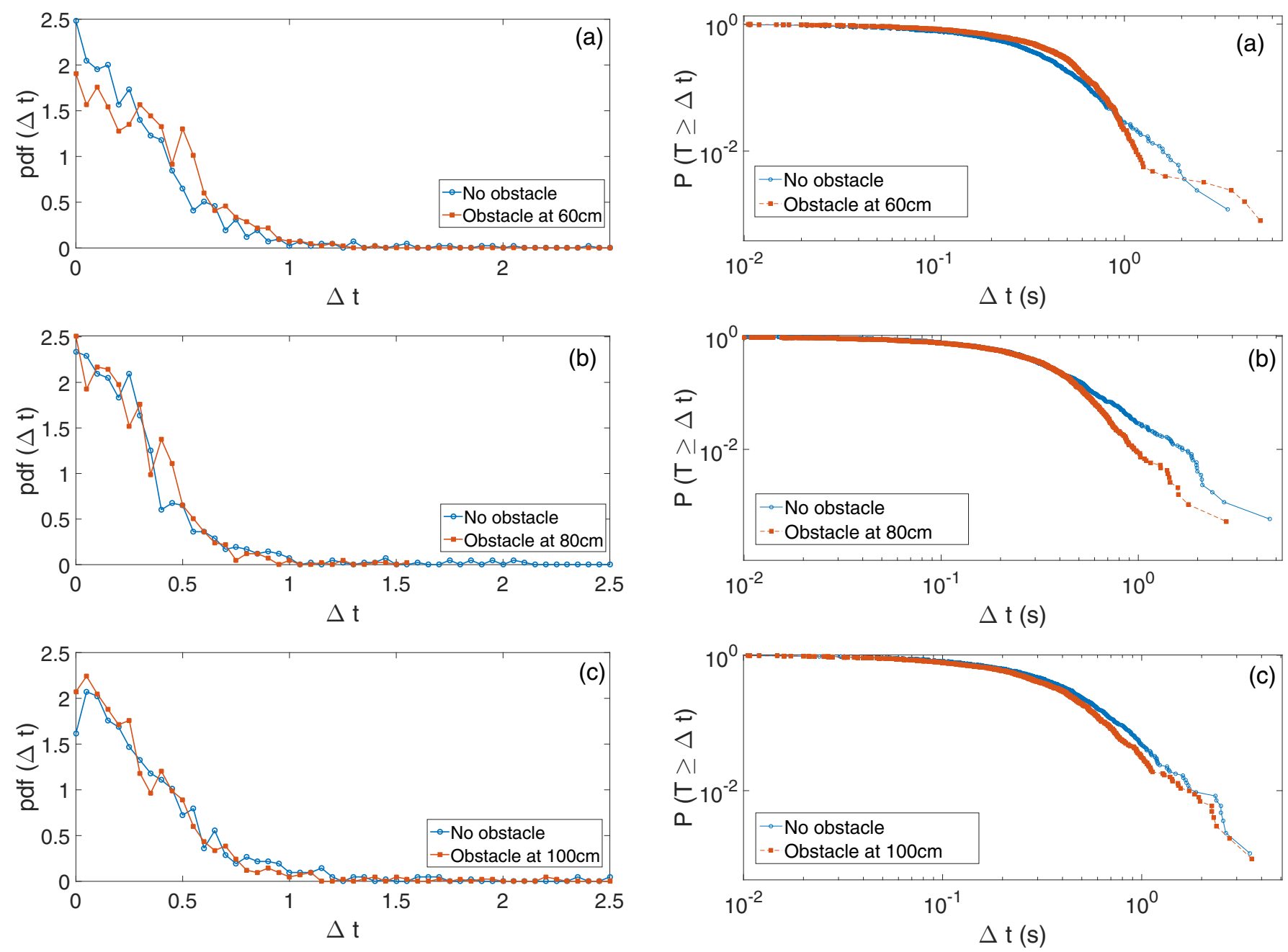

FIG. 4. Probability density function of $\Delta t$ comparing the trials with and without obstacle for (a) herd A, (b) herd B, and (c) herd C. Blue circles and red squares represent, respectively, the case when there is no obstacle and when the obstacle is at the positions indicated in the legends.

placed farther, or when there is no obstacle. In Fig. 6(b) we report an example of a typical clog with the obstacle at $60 \mathrm{~cm}$ (recall that sheep clog mainly at the hip) in comparison with another one for the no obstacle situation [Fig. 6(a)]. A extreme example of this alteration of the clogging nature is reported in Fig. 6(c) where, near the end of one test, we observed one of such obstacle-doorjamb clogs which becomes obvious as there are not sheep on the other side of the obstacle. Considering this change in the clogging nature - that was already reported for the case of grains discharged from silos [25] and in pedestrian simulations [24] — we speculate that the shape of the survival function obtained for the obstacle at $60 \mathrm{~cm}$ can be understood as a combination of the intermittent flows through two independent doors, namely, the passageway between the obstacle and the doorjambs. If that would be true, we can restate the problem in terms of the sheep passage through the two bottlenecks between the obstacle and the two doorjambs [indicated with red lines in Fig. 6(b)] which are, indeed, narrower than the actual door. In order to check the plausibility of this hypothesis we have calculated the distribution which

FIG. 5. Logarithmic plot of the complementary cumulative distribution function of $\Delta t$ comparing the trials with and without obstacle for (a) herd A, (b) herd B, and (c) herd C. Blue circles and red squares represent, respectively, the function when there is no obstacle and when the obstacle is at the positions indicated in the legends. The complementary distribution function is also called survival or reliability function and can be seen as the probability of finding a passage time larger than the values indicated in the horizontal axis.

results from combining the passage times of two independent doors narrower than the actual door. To this end, we have taken the results of $\Delta t$ obtained in previous experiments [30] performed using a door of $77 \mathrm{~cm}$ (a distance which is about the gap existing between the obstacle at $60 \mathrm{~cm}$ and the $96 \mathrm{~cm}$ wide door). These data corresponded to 32 different tests which we split in two groups of 16 , leading to around 1250 sheep passages in each group. Then, we combine the two distributions assuming that the flow through each bottleneck is independent of the other. The new combined distribution is displayed in Fig. 7 together with the survival distribution of the $96 \mathrm{~cm}$ door without obstacle. Admittedly this is a very simplistic view of the problem as, indeed, after crossing these obstacle-doorjamb bottlenecks the sheep must cross the proper door where there is a merging of the flows. Even though the combined distribution displayed in Fig. 7 does not take into account the clogging at the main door (which is 

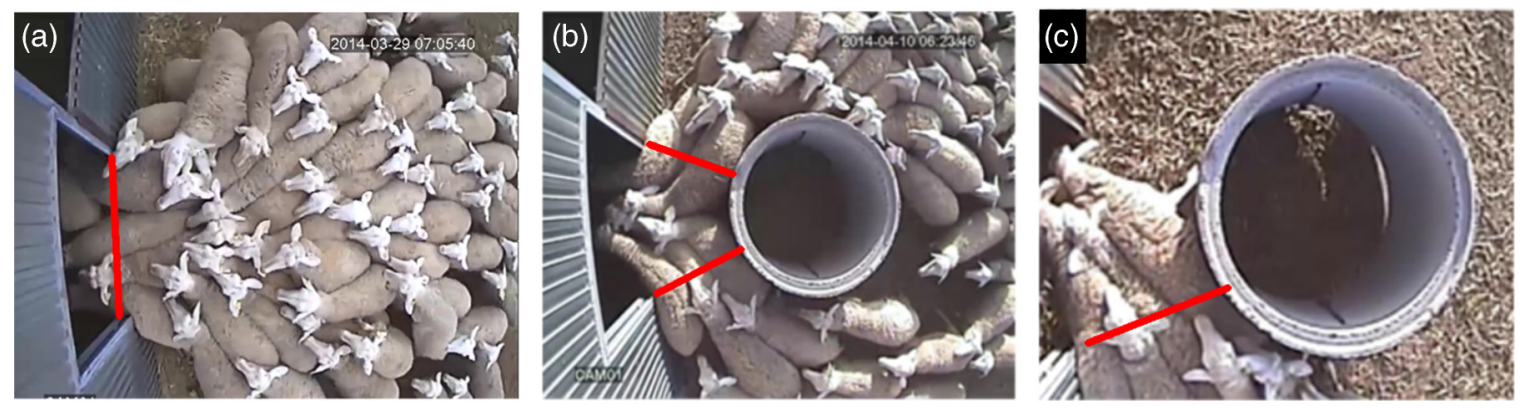

FIG. 6. Pictures illustrating three different types of clogs. Figure (a) represents the typical clog observed when there is no obstacle or when the obstacle is far from the door: the hips of the sheep clog at the very door. Figure (b) shows a situation found when the obstacle is placed at $60 \mathrm{~cm}$ where sheep clog between the obstacle and the doorjamb. In (c) we display a case where the clogging between the obstacle at $60 \mathrm{~cm}$ and the doorjamb becomes evident as it happens in only one side; all the sheep in the other side have already gone into the barn. The lines drawn in (a) and (b) indicate the narrower place through which the animals have to pass in each case. Note that (c) corresponds to a video that was recorded with a different zoom than (a) and (b).

very likely to occur), we observe that the most conspicuous feature of the distribution reported in Fig. 5(a) for the obstacle scenario is qualitatively recovered. Of course, the aim of this representation is just providing a sound interpretation of the surprising results obtained when the obstacle is at $60 \mathrm{~cm}$. A complete quantitative description would require more experiments and, overall, to take into consideration the entire geometry of the system. Indeed, there is a number of aspects that might have an important influence and should therefore be considered, such as the merging of flows after the two bottlenecks [35], the passage through the actual door, and the fact that the flow through inclined gates is not necessarily the same than through horizontal ones (see a granular example in [36,37]).

\section{CONCLUSIONS}

In this work we have reported detailed analysis of the effect that obstacle position has on the sheep flow properties through constrictions. We show, with live beings, the existence of

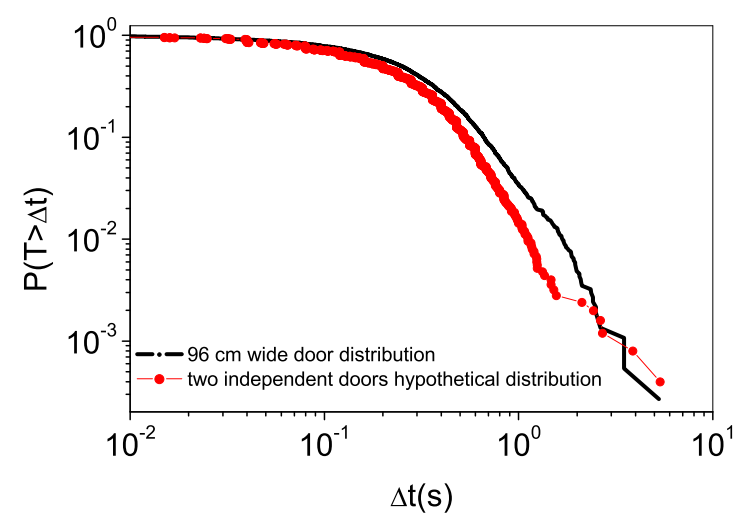

FIG. 7. Logarithmic plot of the complementary cumulative distribution function of $\Delta t$ of two different scenarios. Red dots are used to represent a hypothetical distribution obtained by combining the $\Delta t$ of two sets of 16 evacuations through a $77 \mathrm{~cm}$ wide door. This is done hypothesizing that the passage through both sides of the obstacle can be modeled as an uncorrelated passage through two narrower doors. For comparison with the results of Fig. 6, the black line indicates the distribution of $\Delta t$ obtained for the $96 \mathrm{~cm}$ wide door without obstacle. a nonmonotonous behavior of the flow rate versus obstacle position. Among the locations studied, $80 \mathrm{~cm}$ seems to be the best choice: at a closer position the obstacle is detrimental, while a more distant placement is beneficial but the effect becomes weaker than for the $80 \mathrm{~cm}$ case. Although we cannot assure that $80 \mathrm{~cm}$ is the optimum position, these results point towards the existence of such an optimum distance around this value. At the same time, these outcomes evidence the extreme sensitivity of the flow to the obstacle position stressing the great importance of making a proper choice depending on the situation.

Furthermore, we observe that when the obstacle is too close to the door the distribution of passage times is notably different to the other situations. In particular, we observe a reduction of the probability of finding clogs between 1 and $2 \mathrm{~s}$, followed by an important increase of this probability for larger clogs. We propose that this surprising behavior could be related with an alteration of the nature of clogs as, obviously, when the obstacle is too close to the door, clogging develops between the doorjamb and the obstacle instead of at the proper door. Therefore, the flow rate in this scenario becomes a combination of the flows through both sides of the obstacle. Based on this idea we have proposed a simple model that qualitatively reproduces the features obtained in the distribution function. We expect further investigation of this effect as, up to now, the change in the phenomenology when the obstacle is too close to the door has been only thoroughly studied for the case of inert grains flowing out of a silo. Unfortunately, in the silo case, nothing similar to the sheep behavior can be observed as when there is a clog, it remains stable forever, contrary to what happens with sheep, pedestrians, or active matter in general.

Finally, we report that, in all the scenarios analyzed, the flow rate is constant over the whole process. This result differs from what was recently reported for the case of pedestrians, and suggests either that pressure has a negligible effect on the flow rate, or that pressure remains constant during the entrance procedure. The first hypothesis seems to be incompatible with the proved fact that the obstacle leads to flow improvement, so we think that the second option is more trustworthy. Indeed, looking at the videos it is easy to note the high pressure that sheep make near the gate, but it also seems that the animals at the back of the herd are not pushing at all. Therefore, if 
pressure at the gate is only the result of the contribution of the dozen sheep closest to the door, it follows that the idea of having a constant pressure over the whole process is not as hare-brained as could be thought a priori. If this hypothesis is true, a straightforward implication would be an independence of the flow properties on the size of the herd, which is opposite to what is expected for the human case. Clearly, this idea should be corroborated in the future by experiments with larger number of sheep or by means of numerical simulations that incorporate some kind of short range social force.

\section{ACKNOWLEDGMENTS}

We thank Sergio Ardanza-Trevijano for his useful comments concerning the statistical analysis of our work. We are indebted to Luis Fernado Urrea for technical assistance, and Tomás Yagüe for allowing us to perform these observations at his farm with his sheep. This work was funded by Mutua Montañesa and Ministerio de Economía y Competitividad (Spanish Government) through Project No. FIS2014-57325.
[1] D. Helbing, I. J. Farkas, and T. Vicsek, Simulating dynamical features of escape panic, Nature (London) 407, 487 (2000).

[2] A. Schadschneider, A. Kirchner, and K. Nishinari, in Cellular Automata 2002, edited by S. Bandini, B. Chopard, and M. Tomassini (Springer, Berlin, 2002), p. 239.

[3] A. Kirchner, K. Nishinari, and A. Schadschneider, Friction effects and clogging in a cellular automaton model for pedestrian dynamics, Phys. Rev. E 67, 056122 (2003).

[4] S. P. Hoogendoorn, Pedestrian flow modeling by adaptive control, Transport. Res. Rec.: J. Transport. Res. Board 1878, 95 (2004).

[5] S. P. Hoogendoorn and W. Daamen, Pedestrian behavior at bottlenecks, Transp. Sci. 39, 147 (2005).

[6] A. Nicolas, S. Bouzat, and M. N. Kuperman, Statistical fluctuations in pedestrian evacuation times and the effect of social contagion, Phys. Rev. E 94, 022313 (2016).

[7] N. W. F. Bode, A. J. Wood, and D. W. Franks, Social networks and models for collective motion in animals, Behav. Ecol. Sociobiol. 65, 117 (2011).

[8] A. John, A. Schadschneider, D. Chowdhury, and K. Nishinari, Trafficlike Collective Movement of Ants on Trails: Absence of a Jammed Phase, Phys. Rev. Lett. 102, 108001 (2009).

[9] N. Shiwakoti, M. Sarvi, G. Rose, and M. Burd, Animal dynamics based approach for modeling pedestrian crowd egress under panic conditions, Transp. Res. B 45, 1433 (2011).

[10] S. A. Soria, R. Josens, and D. R. Parisi, Experimental evidence of the "Faster is Slower" effect in the evacuation of ants, Safety Sci. 50, 1584 (2012).

[11] S. Boari, R. Josens, and D. R. Parisi, Efficient egress of escaping ants stressed with temperature, PloS One 8, e81082 (2013).

[12] D. R. Parisi, S. A. Soria, and R. Josens, Faster-is-slower effect in escaping ants revisited: Ants do not behave like humans, Safety Sci. 72, 274 (2015).

[13] N. Gravish, G. Gold, A. Zangwill, M. A. D. Goodismanb, and D. I. Goldman, Glass-like dynamics in confined and congested ant traffic, Soft Matter 11, 6552 (2015).

[14] C. Saloma, G. J. Perez, G. Tapang, M. Lim, and C. P. Saloma, Self-organized queuing and scale-free behavior in real escape panic, Proc. Natl. Acad. Sci. USA 100, 11947 (2003).

[15] P. Lin, J. Ma, T. Liu, T. Ran, Y. Si, and T. Li, An experimental study of the faster-is-slower effect using mice under panic, Physica A: Stat. Mech. Appl. 452, 157 (2016).

[16] D. R. Parisi and C. O. Dorso, Microscopic dynamics of pedestrian evacuation, Physica A: Stat. Mech. Appl. 354, 606 (2005).
[17] D. Helbing, A. Johansson, J. Mathiesen, M. H. Jensen, and A. Hansen, Analytical Approach to Continuous and Intermittent Bottleneck Flows, Phys. Rev. Lett. 97, 168001 (2006).

[18] D. R. Parisi and C. O. Dorso, Morphological and dynamical aspects of the room evacuation process, Physica A: Stat. Mech. Appl. 385, 343 (2007).

[19] K. Suzuno, A. Tomoeda, and D. Ueyama, Analytical investigation of the faster-is-slower effect with a simplified phenomenological model, Phys. Rev. E 88, 052813 (2013).

[20] A. Garcimartín, I. Zuriguel, J. M. Pastor, C. Martín-Gómez, and D. R. Parisi, Experimental evidence of the "Faster Is Slower" effect, Transport. Res. Proc. 2, 760 (2014).

[21] J. M. Pastor, A. Garcimartín, P. A. Gago, J. P. Peralta, C. MartínGómez, L. M. Ferrer, D. Maza, D. R. Parisi, L. A. Pugnaloni, and I. Zuriguel, Experimental proof of Faster-is-slower in multiparticle systems flowing through bottlenecks, Phys. Rev. E 92, 062817 (2015).

[22] D. Helbing, L. Buzna, and A. Johansson, Self-organized pedestrian crowd dynamics: Experiments, simulations, and design solutions, Transport. Sci. 39, 1 (2005).

[23] D. Yanagisawa, A. Kimura, A. Tomoeda, R. Nishi, Y. Suma, K. Ohtsuka, and K. Nishinari, Introduction of frictional and turning function for pedestrian outflow with an obstacle, Phys. Rev. E 80, 036110 (2009).

[24] G. A. Frank and C. O. Dorso, Room evacuation in the presence of an obstacle, Physica A (Amsterdam, Neth.) 390, 2135 (2011).

[25] I. Zuriguel, A. Janda, A. Garcimartín, C. Lozano, R. Arévalo, and D. Maza, Silo Clogging Reduction by the Presence of an Obstacle, Phys. Rev. Lett. 107, 278001 (2011).

[26] F. Alonso-Marroquin, S. I. Azeezullah, S. A. Galindo-Torres, and L. M. Olsen-Kettle, Bottlenecks in granular flow: When does an obstacle increase the flow rate in an hourglass? Phys. Rev. E 85, 020301(R) (2012).

[27] C. Lozano, A. Janda, A. Garcimartín, D. Maza, and I. Zuriguel, Flow and clogging in a silo with an obstacle above the orifice, Phys. Rev. E 86, 031306 (2012).

[28] I. Zuriguel, D. R. Parisi, R. C. Hidalgo, C. Lozano, A. Janda, P. A. Gago, J. P. Peralta, L. M. Ferrer, L. A. Pugnaloni, E. Clément, D. Maza, I. Pagonabarraga, and A. Garcimartín, Clogging transition of many-particle systems flowing through bottlenecks, Sci. Rep. 4, 7324 (2014).

[29] N. Shiwakoti, M. Sarvi, and M. Burd, Using non-human biological entities to understand pedestrian crowd behavior under emergency conditions, Safety Sci. 66, 1 (2014).

[30] A. Garcimartín, J. M. Pastor, L. M. Ferrer, J. J. Ramos, C. Martín-Gómez, and I. Zuriguel, Flow and clogging of a sheep 
herd passing through a bottleneck, Phys. Rev. E 91, 022808 (2015).

[31] P. Lin, J. Ma, T. Liu, T. Ran, Y. Si, and T. Li, An experimental study of the impact of an obstacle before an exit using mice under panic (unpublished).

[32] A. Garcimartín, D. R. Parisi, J. M. Pastor, C. Martín-Gómez, and I. Zuriguel, Flow of pedestrians through narrow doors with different competitiveness, J. Stat. Mech. (2016) 043402.

[33] W. Liao, A. Tordeux, A. Seyfried, M. Chraibi, K. Drzycimski, X. Zheng, and Y. Zhao, Steady state of pedestrian flow in bottleneck experiments, Physica A: Stat. Mech. Appl. 461, 248 (2016).

[34] The measurement of the passage times was checked to be consistent when using several procedures by different people. In particular, we modified the thickness of the line from 1 to
5 pixels, we marked the head or the hip of the sheep, and we moved the line from 0.8 to 1.2 sheep bodies. Obviously, the passage times varied (taking the hip gives rise to slightly higher passage times) but the difference in the passage times between consecutive animals $(\Delta t)$ was smaller than the estimated resolution.

[35] T. Ezaki, D. Yanagisawa, and K. Nishinari, Pedestrian flow through multiple bottlenecks, Phys. Rev. E 86, 026118 (2012).

[36] C. C. Thomas and D. J. Durian, Geometry dependence of the clogging transition in tilted hoppers, Phys. Rev. E 87, 052201 (2013).

[37] A. Medina, D. Cabrera, A. López-Villa, and M. Pliego, Discharge rates of dry granular material from bins with lateral exit holes, Powder Technol. 253, 270 (2014). 